Case Report

\title{
Delays in Diagnosis of Pulmonary Lymphangitic Carcinomatosis due to Benign Presentation
}

\author{
Swati Pandey $\mathbb{D}^{1}$ and Shishir Ojha $\mathbb{D}^{2}$ \\ ${ }^{1}$ Elkhart General Hospital, 600 East Blvd, Elkhart, IN 46514, USA \\ ${ }^{2}$ Elkhart General Hospital, Beacon Medical Group Pulmonology, 500 Arcade Avenue, Suite 210, Elkhart, IN 46514, USA \\ Correspondence should be addressed to Shishir Ojha; ojha_sh@yahoo.com
}

Received 15 May 2019; Accepted 26 October 2019; Published 24 November 2020

Academic Editor: Jose I. Mayordomo

Copyright (c) 2020 Swati Pandey and Shishir Ojha. This is an open access article distributed under the Creative Commons Attribution License, which permits unrestricted use, distribution, and reproduction in any medium, provided the original work is properly cited.

The diagnosis of lymphangitic carcinomatosis is challenging due to the manifestation of nonspecific symptoms and radiographic abnormalities that bear similarity to those of interstitial lung disease. Herein, we report the case of a 53-year-old woman diagnosed with lymphangitic carcinomatosis from metastatic gastric adenocarcinoma, 3 months after her initial presentation.

\section{Case}

A 53-year-old female was referred to the pulmonary clinic due to cough and chest pressure for the past 3 months, during which several chest X-rays and a computed tomography (CT) scan of the chest were taken. She was initially expectorating green sputum; however, the sputum production reduced after she received antibiotics from her primary care physician (PCP). Additionally, she received two courses of steroids from visits to the emergency department, which stabilized her cough. The patient also had occasional symptoms of postprandial bloating.

She was a lifelong nonsmoker, who worked in farms and denied any exposure to molds or pets. During examination, she was afebrile with an oxygen saturation of $87 \%$ on room air inhalation. Her physical exam revealed coarse breath sounds on auscultation and decreased breath sounds at the level of the left lung base posteriorly. A CT scan of her chest (Figure 1) showed bilateral linear coarse reticulations with no peripheral predilection or ground-glass opacities and a small amount of left pleural effusion; the abdomen did not show any abnormality in the CT scan. Based on her symptoms and chest CT findings, we suspected a diagnosis of interstitial lung disease (ILD).
Laboratory findings were negative for antinuclear antibodies and antineutrophil cytoplasmic antibodies. Video-assisted thoracic surgical biopsy and transbronchial cryobiopsy were discussed, and the risks and benefits of each procedure were explained. She chose to undergo a transbronchial cryobiopsy, which was performed 3 months after her initial presentation to PCP. Three cryobiopsy samples were obtained-two from the lateral basal segment and one from the posterior basal segment of the right lower lobe-for pathologic evaluation. Additionally, four forceps biopsy samples were collected-two each from anterior basal and superior segment of the right lower lobe-for microbiological evaluation. The pathology samples were sent to a tertiary care center to be reviewed by a pulmonary pathologist with expertise in ILD.

Based on the morphology, the patient was diagnosed with a tumor that was characterized as metastatic adenocarcinoma with lymphangitic spread (Figures 2 and 3). Tumor cells were positive for keratin and negative for CD31, thus confirming the diagnosis. A second round of histological staining was negative for thyroid transcription factor-1, GATA 3, mammaglobin, and gross cystic disease fluid protein-15 (GCDFP-15), thereby excluding the lungs and breast as primaries. As CDX2 was strongly positive, gastric 


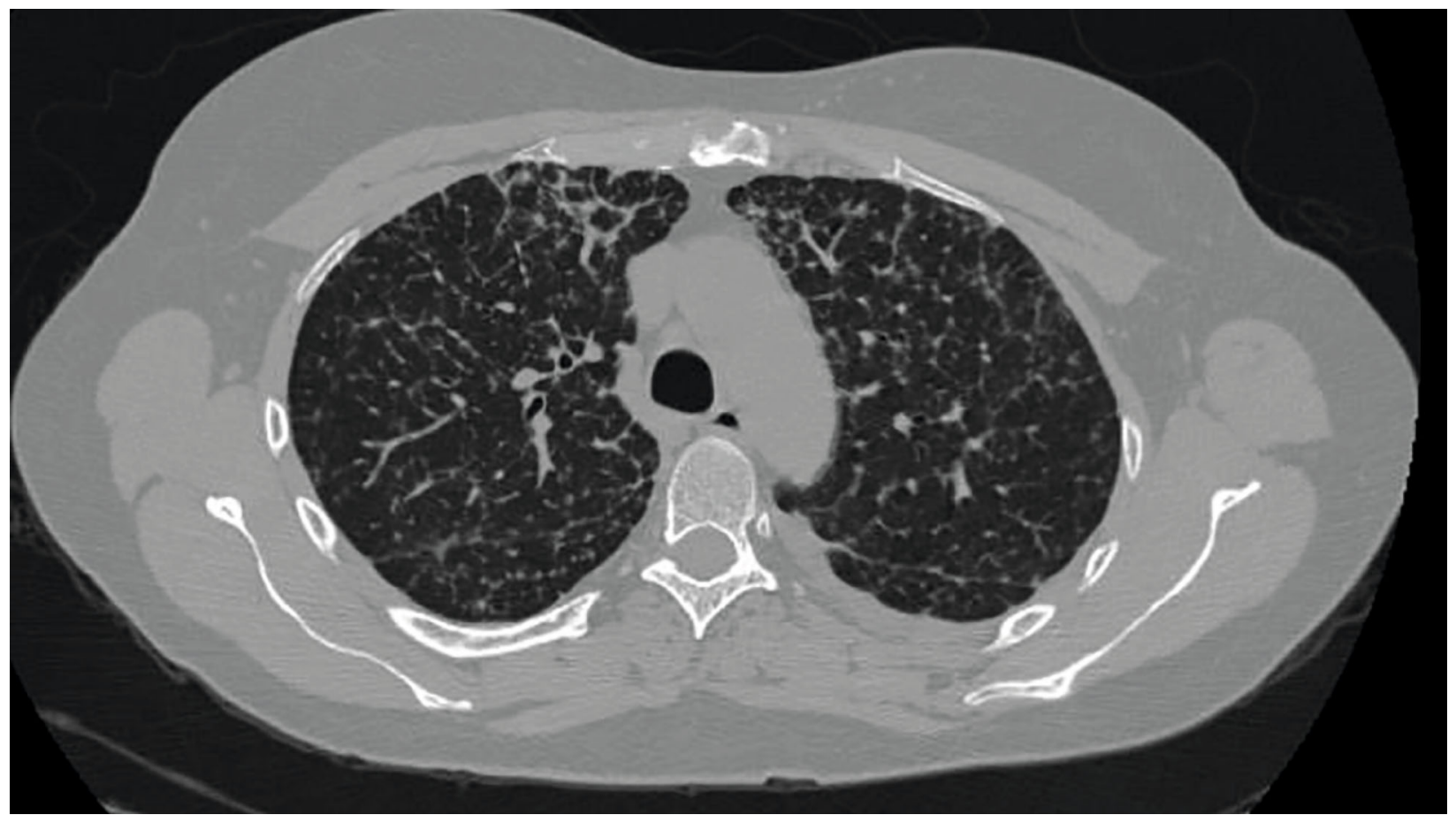

Figure 1: Chest CT scan.

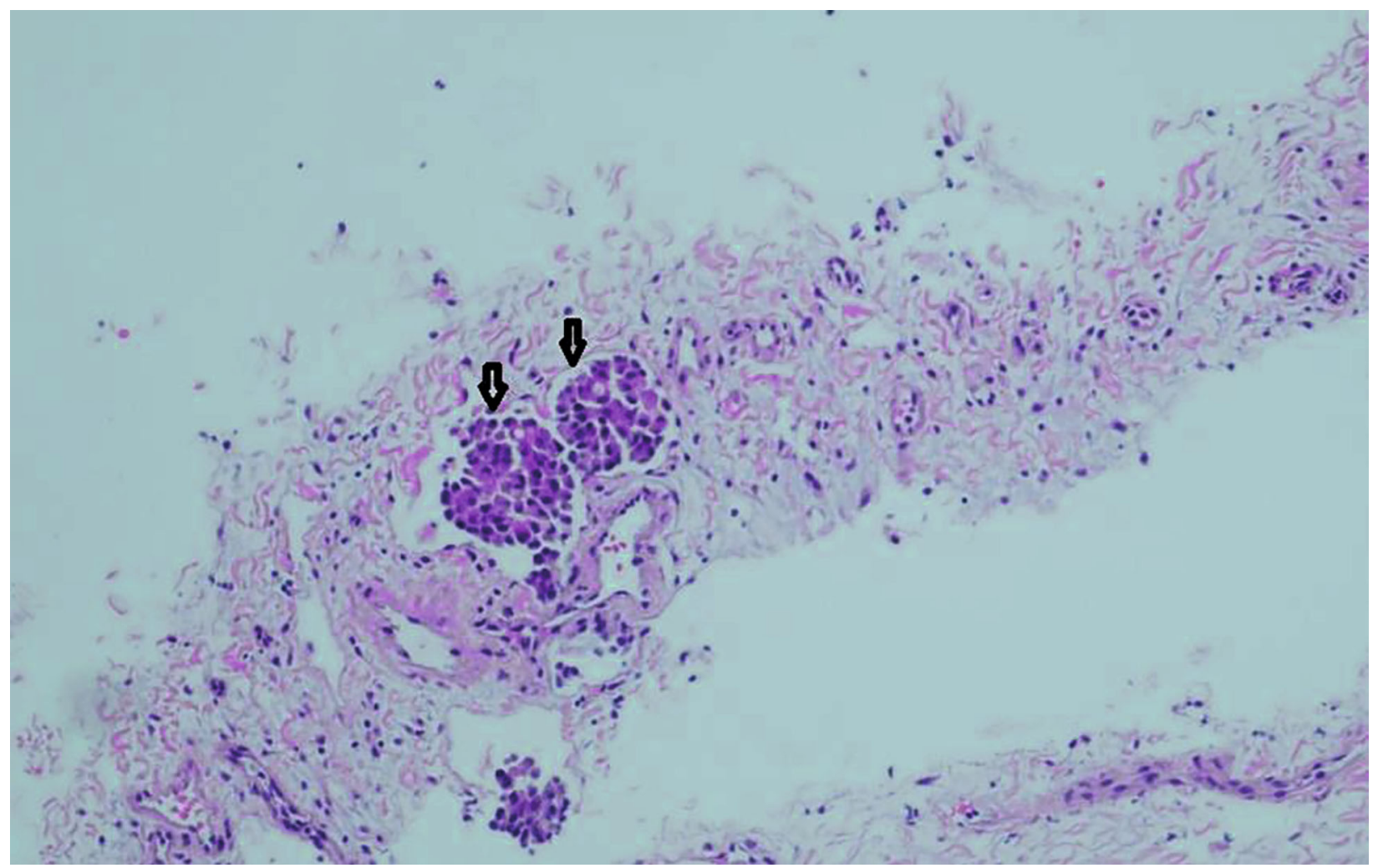

Figure 2: Hematoxylin and eosin-stained slide of cryobiopsy of lung. Arrow pointing to lymph-vascular invasion of tumor cells.

primary was suspected. The differential diagnosis in the upper gastrointestinal primary or pancreatobiliary primary at this stage of staining supported the assumption.
Thereafter, the patient underwent a repeat CT scan of the abdomen and pelvis, which did not reveal any mass or abnormality to suggest a primary. Three weeks after the 


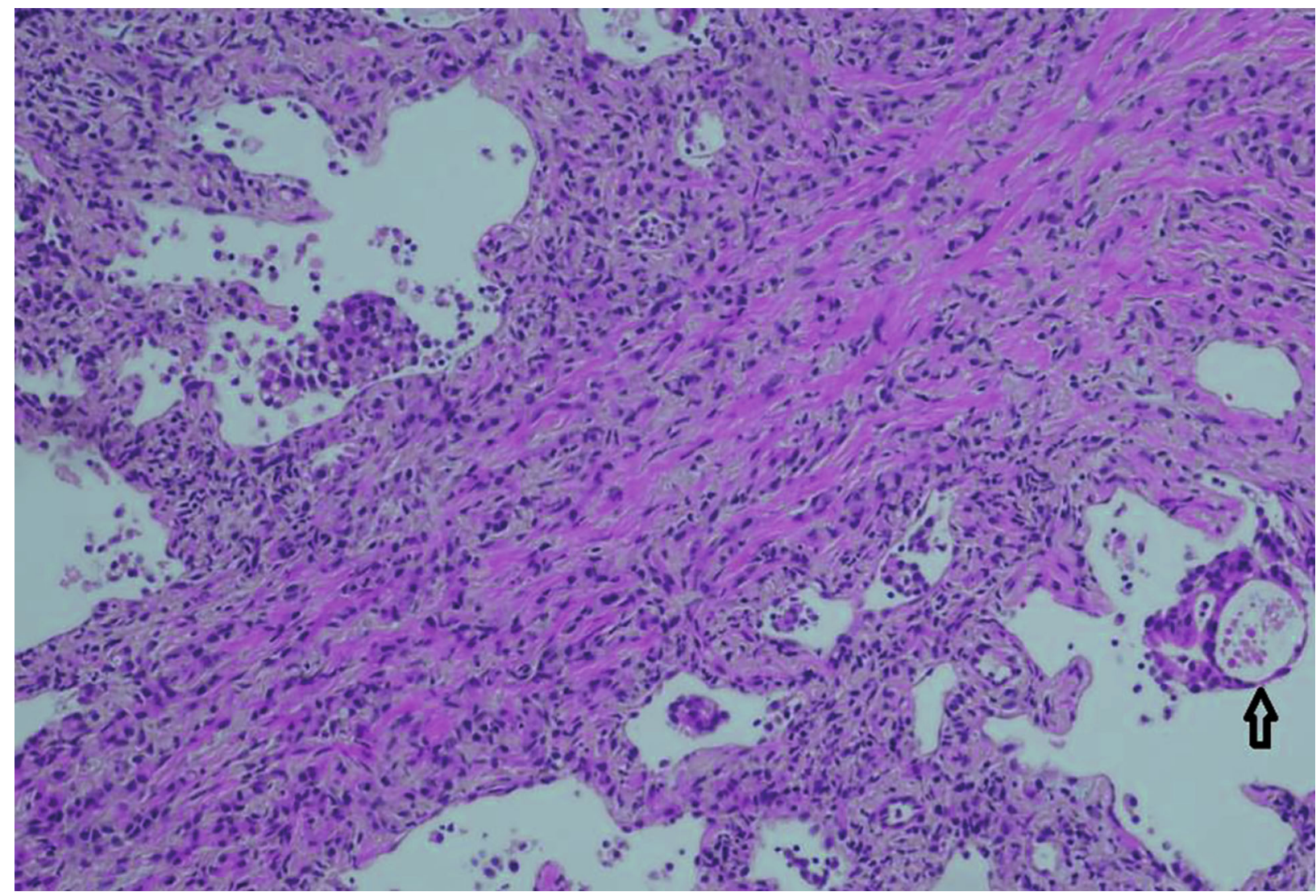

Figure 3: Hematoxylin and eosin-stained slide of cryobiopsy of lung. Arrow pointing to adenocarcinoma.

bronchoscopic biopsy, she underwent an upper endoscopy, which revealed an ulcerated gastric mass occupying the cardia and antrum of the stomach. Biopsies from the mass revealed moderate to poorly differentiated invasive adenocarcinoma with lymphovascular invasion (Figure 4).

Two days after the endoscopic biopsies, the patient was seen by an oncologist in an outpatient setting where several chemotherapy options ranging from aggressive regimens, such as FOLFOX, to the least aggressive $5 \mathrm{FU}$ with leucovorin were discussed. However, the patient declined the treatment. Subsequently, she was hospitalized three times, 2 weeks apart, for worsening dyspnea and was treated with thoracentesis on each occasion. During her last admissions, a chest tube was placed due to iatrogenic pneumothorax after thoracentesis. The patient experienced worsening dyspnea and hypoxemia, and comfort measures were initiated. The patient died 10 days after the last hospitalization, about 2 months after her diagnosis.

\section{Discussion}

We report the case of occult gastric malignancy with pulmonary lymphangitic carcinomatosis (PLC).

Pulmonary metastasis is rare with gastric cancer, representing less than one percent of distant metastasis. The most commonly seen pattern of pulmonary metastasis is hematogenous which accounts for $52.3 \%$, followed by pleural metastasis (35.2\%), and lymphangitic spread is the least common of this rare pattern, accounting for only $26.4 \%$ of pulmonary metastasis [1]. Gastric lesions or abnormality in other viscera were absent on the CT scan of the abdomen. Her chest CT revealed interlobular septal thickening. The absence of mediastinal or hilar lymph node enlargement and lung masses or nodules excluded a diagnosis of malignancy. Despite widespread pulmonary parenchymal abnormalities, there was no lymph node involvement. Mediastinal or hilar lymphadenopathy is commonly seen with lymphangitic spread [2], but lymph node involvement is not essential. The mechanism of lymphangitic spread is hematogenous tumor embolism to the lungs and rarely due to contiguous lymphangitic spread [3].

The diagnosis of metastatic cancer with pulmonary carcinomatosis is often delayed by months from the onset of symptoms and imaging studies. This delay is mainly due to two attributes that are strongly associated with its presentation and imaging studies. First, patient characteristics play an important role. Most patients present with dry cough and dyspnea due to pulmonary parenchymal involvement, irrespective of the origin of the primary. Hence, the workup for the symptoms is performed and does not involve imaging or diagnostic tests, which can reveal the primary. A patient's age is usually less than that of a typical lung cancer patient because a wide variety of cancers that occur at a young age may present with lymphangitic spread. Patients are often nonsmokers, thereby lowering the suspicion for lung cancer. Moreover, patients are often empirically treated with 


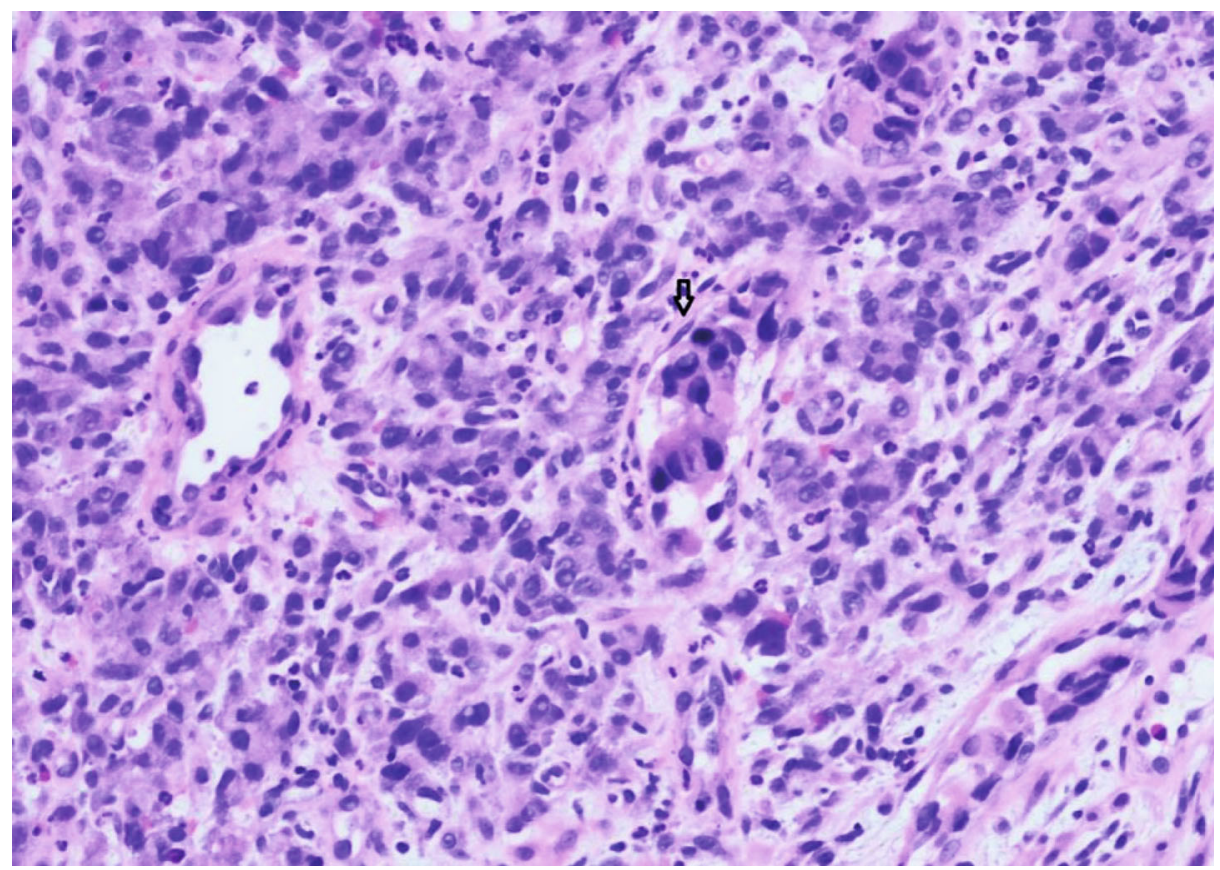

Figure 4: Hematoxylin and eosin-stained slide of Gastric Biopsy: arrow pointing to gastric adenocarcinoma.

antibiotics for presumed respiratory tract infection and inhalers for dyspnea.

Second, a radiologist is often misled by imaging patterns, whether on chest X-ray or chest $\mathrm{CT}$, and the requesting physician may be convinced of an ILD diagnosis. The CT pattern of PLC is very similar to that of many ILDs, including sarcoidosis, which is an arduous task to differentiate. Subtle differences do exist, but these are not easy to spot and include a great involvement of the interlobular septa and interstitium in PLC and more distortion of secondary pulmonary lobule due to fibrosis in sarcoid [4]. Thus, owing to the clinical presentation and radiographic patterns, our focus was on the diagnosis of an underlying primary pulmonary parenchymal disease, specifically an ILD, rather than casting a wider net, which would have included an examination for malignancy.

We performed a literature search using the PubMed database for studies reporting cases of lymphangitic carcinomatosis that were suspected as an ILD by treating physicians. The extracted publications are listed in Table 1 [5-18]. Information on the types of delays that occurred in the case reports are listed (Table 1), which included time interval from the first occurrence of a symptom to the first contact with a physician and the interval from symptoms onset, diagnosis, and presentation to death.

Delays in the diagnosis of primary lung cancer diagnosis range from 7 days to 6 months and from onset of symptoms to contact with a physician [19]. The largest trial that has investigated 380 consecutive patients with primary lung cancer found that the median duration from the onset of symptoms to visit with a physician was 7 days, that from the physician's visit to diagnosis was 31 days, and that from symptom onset to diagnosis was 50 days [20]. As depicted, the wait times are similar to the delays in the diagnosis of primary lung cancer.
The reason for the delay in the diagnosis of PLC can be due to the misinterpretation of the presentation and radiographic abnormality by the treating physician, and such delay reduces patients' duration of survival after diagnosis. Compounding this grave situation is that patients spend their remaining life under intensive care while undergoing invasive procedures to diagnose what was misinterpreted as ILD. The overall 5-year survival rate for lung cancer for all stages is $19.4 \%$ [21]. In comparison, as depicted in the table, several diagnoses of PLC occurred in the intensive care unit on vented patients, and most of them died within a day to few weeks thereafter.

The misinterpretation of PLC as an ILD is more likely to occur if the imaging study shows findings of only pulmonary carcinomatosis, i.e., only interstitial changes, which are very similar to many ILDs, without lung mass or a nodule. Although many ILDs present with lymph node enlargement, the presence of mediastinal or hilar lymph node enlargement raises the suspicion of malignancy.

The patient in this case report did not have mediastinal or hilar lymphadenopathy, unlike most patients with pulmonary carcinomatosis who have lymphadenopathy. If mediastinal or hilar lymphadenopathy were present, then a much more conservative approach, such as biopsy of the lymph node with endobronchial ultrasound with or without transbronchial forceps biopsy, would have been considered. Instead, due to the presentation, a much more invasive approach than needed for the diagnosis of malignancy, such as transbronchial cryobiopsy, was undertaken. Transbronchial cryobiopsy is a new technique that enables pulmonologists to obtain a larger specimen than the traditional transbronchial forceps biopsy [22], but whether it can serve as an alternative to surgical biopsy for the diagnosis of ILDs is a subject of investigation. Nevertheless, the risks of 


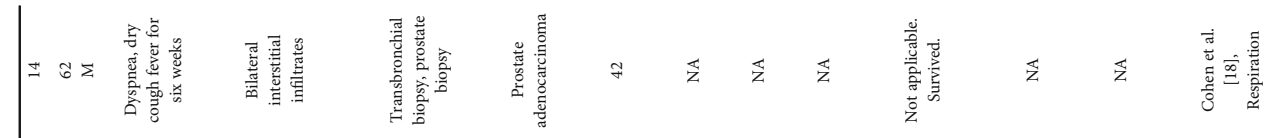

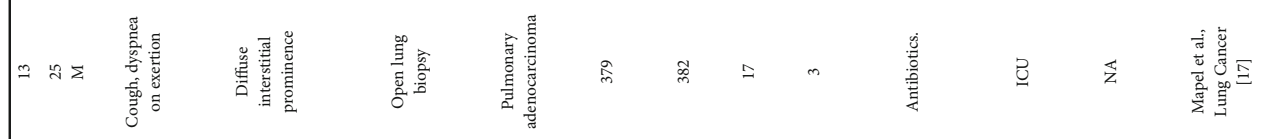

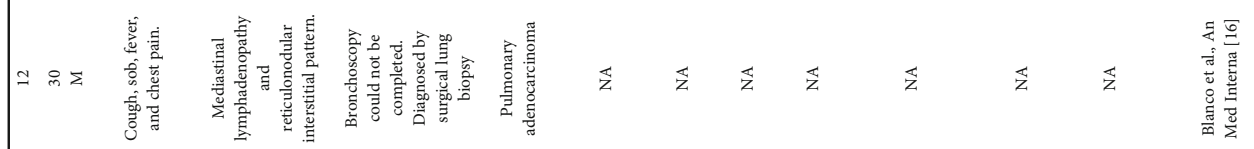

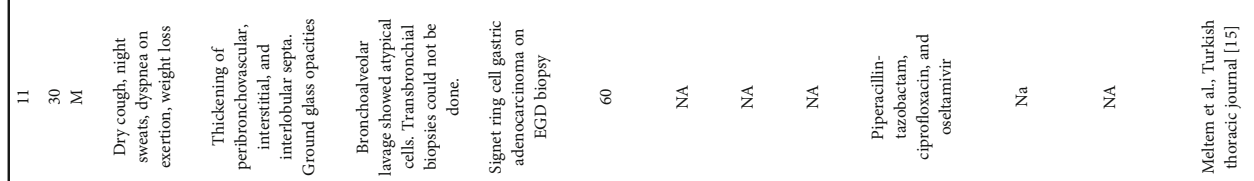

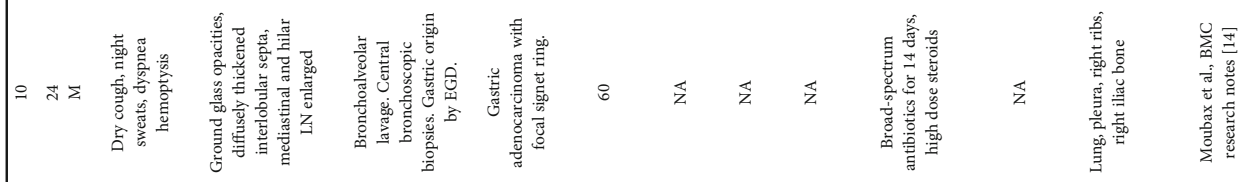

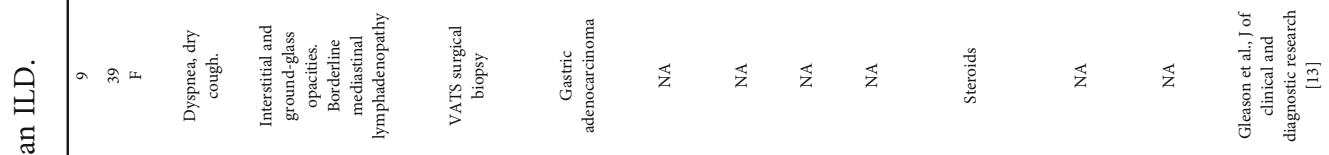

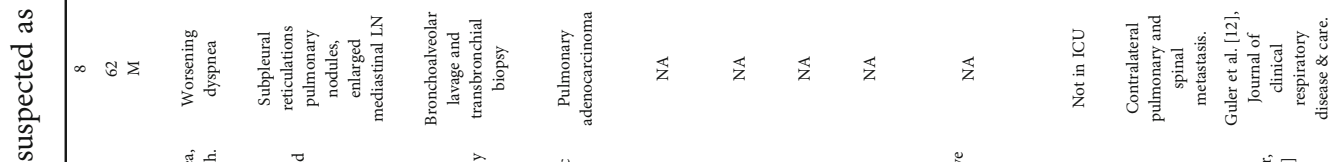

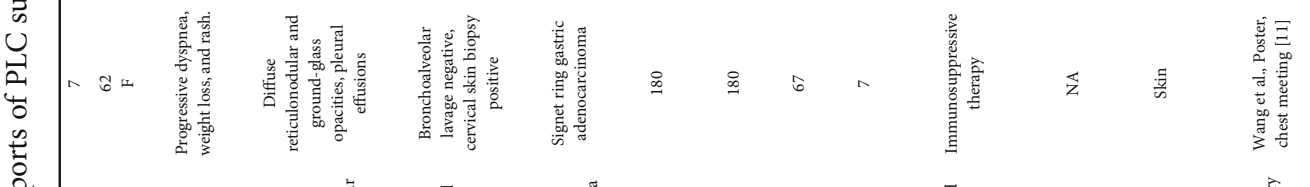

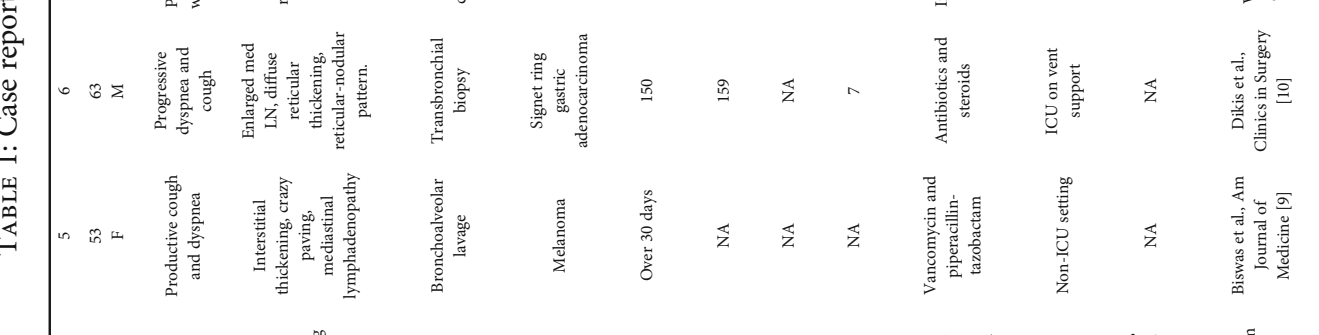

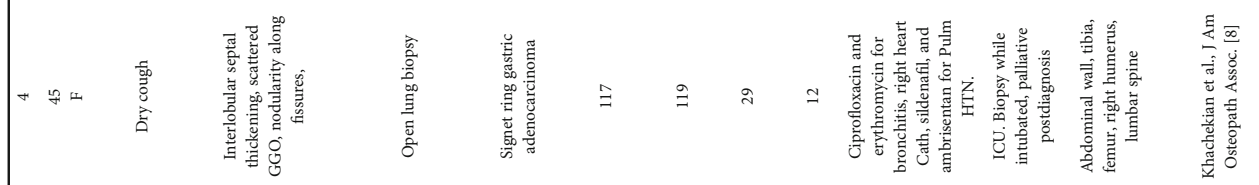

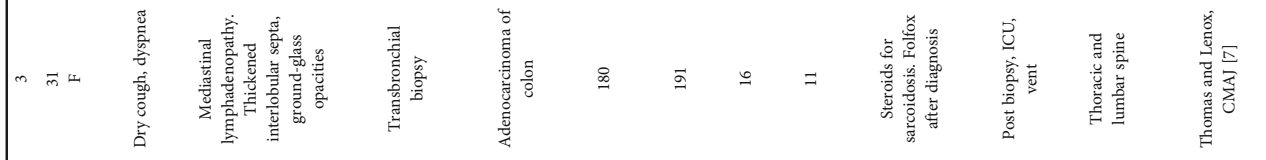

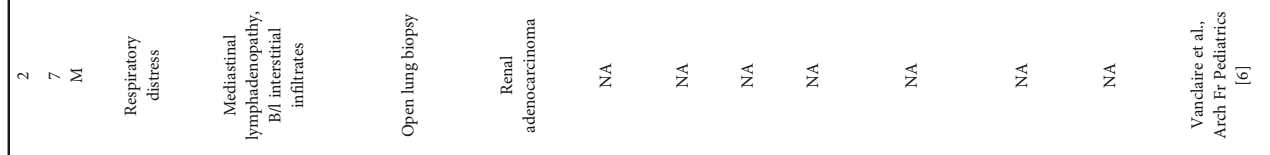

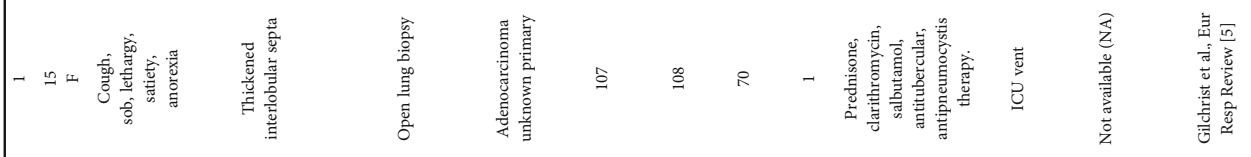

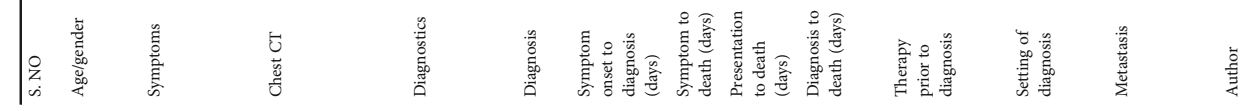


pneumothorax and bleeding are higher than those of forceps biopsy [23], but the overall morbidity is lower than that of surgical lung biopsy [23]. Therefore, patients with PLC undergo more invasive biopsies than needed to obtain a diagnosis, such as surgical lung biopsies, due to the resemblance to ILD in CT scans. However, the limited therapeutic options available for ILDs prevent many patients from undergoing a diagnostic biopsy as the diagnostic procedures are considered to be too risky by their pulmonologist [24]. Therefore, this practice can place patients at the risk of not getting diagnosed for a lethal condition while getting empirically treated for ILD.

ILD is more prevalent than PLC. Therefore, due to the similarity in presentation, clinicians will first seek and investigate the diagnosis of ILD [25] [26], hence being misled. Interestingly, the misdiagnosis of PLC occurs with an even less common disease entity, i.e., Erdheim-Chester disease, which is presented as PLC [27]. Erdheim-Chester disease is a rare disease listed under National Organization for Rare Disorders. Thus, there is precedence for the misdiagnosis of PLC, not only with ILD but also other conditions, warranting robust investigations to enable definite conclusions to be made.

\section{Conflicts of Interest}

The authors declare that they have no conflicts of interest.

\section{Acknowledgments}

The pictures of pathology slides were provided by the Department of Pathology, Benefis Medical Center, Great Falls, MT.

\section{References}

[1] J. H. Kong, J. Lee, C.-A. Yi et al., "Lung metastases in metastatic gastric cancer: pattern of lung metastases and clinical outcome," Gastric Cancer, vol. 15, no. 3, pp. 292-298, 2012.

[2] T. Johkoh, J. Ikezoe, N. Tomiyama et al., "CT findings in lymphangitic carcinomatosis of the lung: correlation with histologic findings and pulmonary function tests," American Journal of Roentgenology, vol. 158, no. 6, pp. 1217-1222, 1992.

[3] E. Dinkel, E. Meyer, A. Mundinger, A. Helwig, U. Blum, and G. Würtemberger, "Interstitial cancerous lung diseases. Lymphangiosis carcinomatosa and leukemic pulmonary infiltrates," Der Radiologe, vol. 30, no. 12, pp. 591-597, 1991.

[4] C. Bergin, V. Roggli, C. Coblentz, and C. Chiles, "The secondary pulmonary lobule: normal and abnormal CT appearances," American Journal of Roentgenology, vol. 151, no. 1, pp. 21-25, 1988.

[5] F. J. Gilchrist, H. Alton, M.-A. Brundler, L. Edwards, A. Plunkett, and S. Rao, "Pulmonary lymphangitic carcinomatosis presenting as severe interstitial lung disease in a 15-yearold female," European Respiratory Review, vol. 20, no. 121, pp. 208-210, 2011.

[6] J. Vanclaire, E. Bodart, P. Schlesser, N. Francotte, G. Thiry, and H. Hainaut, "Pulmonary carcinomatous lymphangitis and renal adenocarcinoma," Archives françaises de pédiatrie, vol. 47, pp. 735-736, 1991.
[7] A. Thomas and R. Lenox, "Pulmonary lymphangitic carcinomatosis as a primary manifestation of colon cancer in a young adult," Canadian Medical Association Journal, vol. 179, no. 4, pp. 338-340, 2008.

[8] A. Khachekian, S. Shargh, and S. Arabian, "Pulmonary lymphangitic carcinomatosis from metastatic gastric adenocarcinoma: case report," The Journal of the American Osteopathic Association, vol. 115, no. 5, pp. 332-337, 2015.

[9] A. Biswas, S. Lulu, and P. S. Sriram, "The great masquerader strikes again!," The American Journal of Medicine, vol. 130, no. 7, pp. e303-e304, 2017.

[10] Ö. Dikis, S. Dulger, T. Yildiz, and S. Atahan, "Signet ring cell gastric carcinoma case diagnosed with pulmonary lymphangitic carcinomatosis," Clinics in Surgery, vol. 2, no. 1676, p. 2, 2017.

[11] "Pulmonary lymphangitic carcinomatosis as a primary manifestation of gastric signet ring cell-type adenocarcinoma in a middle-aged woman: a case report," May 2019, https:// insights.ovid.com/chest/chst/2016/04/001/pulmonarylymphangitic-carcinomatosis-primary/268/00002953.

[12] G. S and B. S, "When malignancies mimic interstitial lung disease: a case series and review of the literature," Journal of Clinical Respiratory Diseases and Care, vol. 1, no. 1, 2015.

[13] J. B. Gleason, J. Dolan, and A. Hadeh, "Lymphangitic pulmonary metastasis: a rare finding in gastric carcinoma," Journal of Clinical and Diagnostic Research, vol. 11, no. 5, p. OJ01, 2017.

[14] K. Moubax, W. Wuyts, V. Vandecaveye, and H. Prenen, "Pulmonary lymphangitic carcinomatosis as a primary manifestation of gastric carcinoma in a young adult: a case report and review of the literature," BMC Research Notes, vol. 5, no. 1, p. 638, 2012.

[15] M. Agca, F. Tokgoz Akyil, M. Hormet et al., "A rare case of progressive dyspnea and bilateral lung infiltration in a young male," Turkish Thoracic Journal, vol. 18, no. 3, pp. 96-99, 2017.

[16] S. Casallo Blanco, F. Marcos Sánchez, S. L. de Matías, A. Viana Alonso, J. Celdrán Gil, and C. E. Núñez, "Lung adenocarcinoma feigning an interstitial lung disease in a 30-year-old man," Anales de medicina interna (Madrid, Spain: 1984), vol. 24, no. 6, pp. 289-291, 2007.

[17] D. W. Mapel, R. H. Fei, and R. E. Crowell, “Adenocarcinoma of the lung presenting as a diffuse interstitial process in a 25 year-old man," Lung Cancer, vol. 15, no. 2, pp. 239-244, 1996.

[18] O. Cohen, L. Leibovici, and A. I. Wysenbeek, "Carcinoma of the prostate presenting as interstitial lung disease," Respiration, vol. 51, no. 2, pp. 158-160, 2004.

[19] A. R. Jensen, J. Mainz, and J. Overgaard, "Impact of delay on diagnosis and treatment of primary lung cancer," Acta Oncologica, vol. 41, no. 2, pp. 147-152, 2009.

[20] GIVIO (Interdisciplinary Group for Cancer Evaluation), "Diagnosis and first-line treatment of patients with lung cancer in Italian general hospitals," Tumori Journal, vol. 75, no. 2, pp. 163-167, 1989.

[21] Cancer of the Lung and Bronchus-Cancer Stat FactsSEERMay 2019, https://seer.cancer.gov/statfacts/html/lungb.html.

[22] R. J. Lentz, A. C. Argento, T. V. Colby, O. B. Rickman, and F. Maldonado, "Transbronchial cryobiopsy for diffuse parenchymal lung disease: a state-of-the-art review of procedural techniques, current evidence, and future challenges," Journal of Thoracic Disease, vol. 9, no. 7, pp. 2186-2203, 2017. 
[23] C. Ravaglia, M. Bonifazi, A. U. Wells et al., "Safety and diagnostic yield of transbronchial lung cryobiopsy in diffuse parenchymal lung diseases: a comparative study versus videoassisted thoracoscopic lung biopsy and a systematic review of the literature," Respiration, vol. 91, no. 3, pp. 215-227, 2016.

[24] Q. Luo, Q. Han, X. Chen, J. Xie, L. Wu, and R. Chen, “The diagnosis efficacy and safety of video-assisted thoracoscopy surgery (VATS) in undefined interstitial lung diseases: a retrospective study," Journal of Thoracic Disease, vol. 5, no. 3, pp. 283-288, 2013.

[25] D. B. Coultas, R. E. Zumwalt, W. C. Black, and R. E. Sobonya, "The epidemiology of interstitial lung diseases," American Journal of Respiratory and Critical Care Medicine, vol. 150, no. 4, pp. 967-972, 1994.

[26] M. Sakuma, S. Fukui, M. Nakamura et al., "Cancer and pulmonary embolism: thrombotic embolism, tumor embolism, and tumor invasion into a large vein," Circulation Journal, vol. 70, no. 6, pp. 744-749, 2006.

[27] S.-A. Yahng, H. H. Kang, S. K. Kim et al., "Erdheim-Chester disease with lung involvement mimicking pulmonary lymphangitic carcinomatosis," The American Journal of the Medical Sciences, vol. 337, no. 4, pp. 302-304, 2009. 